\title{
A Tríplice Hélice e a Construção de Ambientes de Inovação 0 Caso da Incubadora Tecnológica de Luzerna/SC
}

\author{
Fernando Fantoni Bencke ${ }^{1}$ \\ Eric Charles Henri Dorion² \\ Pelayo Munhoz Olea ${ }^{3}$ \\ Cleber Cristiano Prodanov ${ }^{4}$ \\ Fábio Lazzarotti ${ }^{5}$ \\ Lucas Bonacina Roldan ${ }^{6}$
}

http://dx.doi.org/10.21527/2237-6453.2018.43.609-639

Recebido em: 17/11/2015

Aceito em: 31/5/2017

\section{Resumo}

0 presente artigo teve por objetivo analisar os fatores que determinaram a constituição de um ambiente de inovação, o caso da Incubadora Tecnológica de Luzerna (ITL), e o papel desempenhado pelos atores da tríplice hélice. Trata-se de uma pesquisa qualitativa, exploratória e descritiva, que utilizou entrevistas semiestruturadas e análise de conteúdo como principais fontes de coleta e análise de dados. Como abordagem teórica, utilizou-se o modelo da tríplice hélice de Etzkowitz (2009). Os principais resultados

1 Doutor em Administração pela Pontifícia Universidade Católica do Rio Grande do Sul e Universidade de Caxias do Sul. Professor da Universidade do Oeste de Santa Catarina. fernando.bencke@unoesc.edu.br

2 Doutor em Administração pela Université de Sherbrooke, Canadá. Professor da Universidade de Caxias do Sul. echdorion@gmail.com

3 Doutor em Administração e Direção de Empresas pela Universitat Politècnica de Catalunya, Espanha. Professor da Universidade de Caxias do Sul. pelayo.olea@gmail.com

4 Doutor em História Social pela Universidade de São Paulo. Professor da Universidade Feevale. prodanov@feevale.br

5 Doutor em Administração e Turismo pela Universidade do Vale do Itajaí. Professor da Universidade do Oeste de Santa Catarina. fabio.lazzarotti@unoesc.edu.br

6 Doutor em Administração pela Pontifícia Universidade Católica do Rio Grande do Sul e Universidade de Caxias do Sul. Professor da Pontifícia Universidade Católica do Rio Grande do Sul. lucas.roldan@pucrs.br 
destacam os seguintes fatores determinantes no surgimento da ITL: envolvimento de lideranças locais; a iniciativa do poder público municipal; presença de instituições públicas e privadas; inexistências de incubadoras em municípios próximos e a necessidade de criar novas matrizes econômicas à região. Entre o papel dos atores, destaca-se 0 ator governo, em especial a prefeitura de Luzerna, em criar incentivos legais e financeiros, estimular a interação entre diferentes atores, gerar novas demandas de mercado e estimular o surgimento da ITL; 0 ator universidade pela pré-incubadora existente, gerar conhecimento e formar profissionais; 0 ator empresa, pela colaboração interna das incubadas na ITL e a instalação da empresa âncora como incentivo para atrair novos empreendimentos.

Palavras-chave: Tríplice hélice. Ambiente de inovação. Incubadora.

\title{
THE TRIPLE HELIX AND THE CONSTRUCTION OF INNOVATION ENVIRON- MENTS: THE CASE OF THE TECHNOLOGY INCUBATOR OF LUZERNA/SC
}

\begin{abstract}
The objective of this article was to analyze the factors that determined the creation of an innovation environment, the case of the Technological Incubator of Luzern (ITL), and the role played by the actors of the triple helix. It is a qualitative, exploratory and descriptive research that used semi-structured interviews and content analysis as the main sources of data collection and analysis. As a theoretical approach, the triple helix model was used. The main results highlight the following determining factors: involvement of local leaders; the initiative of the municipal public power; presence of public and private institutions; lack of incubators in nearby municipalities; and the need to create new economic matrices for the region. Within the role of the actors, the government actor, especially the Luzern Municipal Government, is responsible for creating legal and financial incentives, stimulating the interaction between different actors, generating new market demands, and stimulating the emergence of ITL. The university actor by the existing pre-incubator, generate knowledge and train professionals. The company actor, for the internal collaboration of incubated in ITL and the installation of the anchor company as an incentive to attract new ventures.
\end{abstract}

Keywords: Triple helix. Innovation environment. Incubator. 
Conforme Etzkowitz (2003, 2009), a inovação tem assumido um significado amplo nos últimos anos. Mais do que o desenvolvimento de novos produtos nas empresas, ela está relacionada à criação de novos arranjos entre as esferas institucionais que promovem as condições para inovação. A transformação e as inter-relações das esferas institucionais da academia, indústria e governo passam a moldar a dinâmica da inovação para o desenvolvimento regional.

O papel da geração e adoção de conhecimento em processos de inovação tem se tornado emergencial na chamada economia baseada no conhecimento. A tríplice hélice pode ser considerada um modelo de sistema de inovação e de desenvolvimento regional baseado no conhecimento e na aprendizagem, concebido como um conjunto de dinâmicas multilineares com base em paradigmas tecnológicos alternativos (ETZKOWITZ, 2009).

A criação de locais, especialmente em âmbito regional, em que os representantes de esferas, tanto públicas quanto privadas, interagem e planejam novas iniciativas para a renovação econômica, social e tecnológica, podem se tornar alternativas de desenvovimento de novas potencialidades regionais, reforçando a importância da interação entre diferentes atores para o fomento da inovação. O movimento de incubadoras surgiu no Brasil na década de 80 , motivado por pesquisadores acadêmicos que buscavam uma alternativa de aplicar suas pesquisas e incentivar a criação de empresas de alta tecnologia. O conceito de incubadora foi difundido pela sociedade brasileira e passou a ser utilizado por governos locais, associações industriais e Organizações Não Governamentais (ETZKOWITZ, 2009).

De acordo com Etzkowitz et al. (2008), a incubadora tornou-se exemplo de atividades da tríplice hélice no Brasil, uma adaptação organizacional que internalizou as relações entre as esferas institucionais, criando um espaço de interação e um ambiente de desenvolvimento da educação empreendedora (ETZKOWITZ et al., 2008; ETZKOWITZ, 2009). 
A Incubadora Tecnológica de Luzerna (ITL), objeto de pesquisa do presente artigo, está localizada no município de Luzerna, no meio-oeste catarinense. Inaugurada em 2010, por meio de uma iniciativa do poder público municipal, com apoio da Fundação de Amparo à Pesquisa e a Inovação do Estado de Santa Catarina (Fapesc), é considerada a única incubadora pública do Estado de Santa Catarina (INCUBADORA..., 2014).

Características e peculiaridades do contexto local, regional, e instituições envolvidas, tornam-se determinantes na constituição ambientes de inovação (AUDY, 2014). Ambientes de inovação constituem-se de maneiras diferentes e sofrem adaptações para se adequar às distintas realidades e peculiaridades locais e nacionais (VEDOVELLO; JUDICE; MACULAN, 2006), o que dificulta a sistematização e replicação de modelos válidos para a constituição de novos empreendimentos (ZOUAIN, 2003).

Se a complexidade do contexto local e nacional interfere na constituição de ambientes de inovação, e o modelo da tríplice hélice que propõe uma aliança entre os atores universidade, empresa e governo, pode se apresentar como um modelo limitado e genérico diante dessas dinâmicas de inovação (SCHOONMAKER; CARAYANNIS, 2013), estudos empíricos tornam-se necessários para buscar compreender como se constituem ambientes de inovação. Nesse contexto, o artigo busca respostas ao seguinte problema: Quais foram os fatores determinantes na constituição da Incubadora Tecnológica de Luzerna (ITL)? O objetivo deste texto foi identificar os fatores que determinam a constituição da Incubadora Tecnológica de Luzerna e explorar o papel desempenhado pelos atores universidade, governo e empresa.

Buscar compreender o papel assumido por diferentes atores é importante à medida que fornece elementos que possam ser úteis para outros ambientes e contextos, como, também, estudar a viabilidade do modelo tríplice hélice em regiões que careçam de novas alternativas de desenvolvimento sustentável. 
Na próxima seção será abordado sobre o modelo da tríplice hélice, papel a ser desempenhado pelos atores universidade, governo e empresa, e incubadoras, seguido pelos procedimentos metodológicos adotados, análise dos dados, discussão dos principais resultados e as conclusões da pesquisa.

\section{Modelo Tríplice Hélice}

A inovação no contexto de desenvolvimento regional resulta do encontro de potencialidades localizadas, até então inexploradas, com iniciativa proativa de atores públicos e privados, em favor da melhoria da competitividade empresarial. A promoção do desenvolvimento regional depende da ação coordenada pelos atores, recursos utilizados e modalidades de coordenação. Trata-se do resultado do esforço dos atores locais na formulação de políticas territoriais capazes de tornar a região o sujeito do seu próprio processo de desenvolvimento (FAURÉ; HASENCLEVER, 2007).

Em um desenvolvimento endógeno, atores adotam estratégias a partir do potencial existente no território e controlam o processo de transformação econômica e social visando ao bem-estar da sociedade. Há o inter-relacionamento entre os recursos locais para formar o seu próprio potencial de desenvolvimento (VÁZQUEZ, 2001). De acordo com Leydesdorff e Etzkowitz (1998), as condições locais fornecem recursos que permitem os arranjos institucionais operarem em rede, possibilitando alianças estratégicas mútuas em busca da inovação.

O modelo da tríplice hélice é conhecido como uma abordagem que propõe um modelo de sistema de inovação para o desenvolvimento regional, constituído do espaço de conhecimento, consenso e aprendizagem, formado por processos de interação em redes, que buscam preencher as lacunas esquecidas pelas políticas públicas (JOHNSON; EDQUIST; LUNDVALL, 2003). 
A abordagem da tríplice hélice foi desenvolvida por Etzkowitz e Leydesdorff (1996) e caracteriza a dinâmica da inovação num contexto de evolução e interação. Os autores defendem a colaboração crescente entre as esferas pública, privada e acadêmica para o desenvolvimento do conhecimento e da inovação. O modelo parte de uma interação que se movimenta como uma hélice tripla, vinculando as três esferas para promover processos de inovação, em que os recursos necessários para formação e operacionalização são fornecidos a partir das condições locais (ETZKOWITZ, 1997). Os recursos existentes influenciam diretamente a capacidade regional em criar nichos de inovação tecnológica, a fim de garantir um espaço na economia global (ETZKOWITZ et al., 2008).

As interações entre universidade, indústria e governo, tese central da tríplice hélice, segundo Etzkowitz (2003, p. 295), "são a chave para melhorar as condições para inovação numa sociedade baseada no conhecimento". A interação entre os atores é a base estratégica para o desenvolvimento social e econômico nas sociedades industriais desenvolvidas e em desenvolvimento. A tríplice hélice possibilita a compreensão dos processos de inovação no seu sentido mais amplo, a universidade por intermédio do conhecimento e transferência de novas tecnologias, a indústria com a produção e prática e o governo financiando e minimizando as dificuldades para o estabelecimento da cultura de inovação e de desenvolvimento (ETZKOWITZ, 2009).

O modelo da tríplice hélice é caracterizado como uma perspectiva evolucionária em que as relações estão em constante transformação, mostrando que as fronteiras são flexíveis e a ação de um dos atores influencia a ação de outros (ETZKOWITZ, 2009). Essas transformações determinam os papéis, antes considerados como competidores, redefinindo à medida que as esferas se intersectam e passam a funcionar em conjunto, cada uma potencializando a ação das outras (LEYDESDORFF, 2005).

Conforme Etzkowitz (2005), as esferas institucionais podem exercer seus papéis especializados num processo de organização regional e, também, de outras esferas, quando uma delas tem sua participação restrita. Quanto aos 
seus papéis tradicionais, Etzkowitz (2009) menciona a indústria como o lócus de produção, o governo como fonte de relações contratuais que garantam interações estáveis e câmbios entre as esferas, e a universidade como fonte de novos conhecimentos e tecnologias.

$\mathrm{Na}$ abordagem sistêmica de inovação, o modelo da tríplice hélice, de Etzkowitz e Leydesdorff (1996), contrapõe-se à tradição schumpeteriana, que associa as inovações às empresas e aos empreendedores, e ao modelo linear de inovação, atribuindo às múltiplas interações entre universidade, empresa e governo como propulsores da inovação e do desenvolvimento no âmbito regional (MEYER; SINILÄINEN; UTECHT, 2013). O modelo representa uma ruptura radical em relação à transferência convencional de tecnologia e conhecimento, pois exige novos arranjos intra e interorganizacionais, os quais requerem um considerável volume de recursos e necessitam de tempo para se desenvolver. O mérito do modelo ocorre pela capacidade de criar as condições para gerar, compartilhar e disseminar o conhecimento apropriado à inovação e aprendizagem, necessários ao desenvolvimento (SAAD; ZAWDIE; MALAIRAJA, 2008).

\section{O Papel da Universidade, do Governo e da Empresa}

A adaptação do modelo de tríplice hélice para a realidade dos países em desenvolvimento e regiões menos favorecidas requer uma ampliação do conceito de universidade (ETZKOWITZ; MELLO; ALMEIDA, 2005). Segundo Etzkowitz (2009), o surgimento da universidade empreendedora pode ser explicado como uma resposta à crescente importância do conhecimento nos sistemas nacionais e regionais de inovação e o reconhecimento de que a universidade é um agente de transferência de conhecimento e tecnologia. Ainda, segundo o autor, os governos, em praticamente todas as partes do mundo, estão se concentrando sobre o potencial da universidade como um recurso para melhorar ambientes de inovação e criar um espaço de desenvolvimento baseado na ciência. 
A universidade empreendedora assume uma postura proativa, colocando o conhecimento em uso, operando num modelo interativo de inovação (ETZKOWITZ, 2013), transformando o conhecimento gerado na agregação de valor econômico e social. Conforme Etzkowitz (2002), a universidade passa por uma dupla transformação: incluir, em sua missão, o papel de desenvolvimento econômico e social e a formação cultural da reprodução da pesquisa com foco organizacional. A tese entre a relação dos atores da tríplice hélice é proposta como uma estratégia de desenvolvimento para preencher as lacunas existentes de capital social e de tecnologia.

Uma universidade empreendedora mantém o seu papel acadêmico tradicional de reprodução social e difusão do conhecimento, situando-os num contexto mais amplo para promoção da inovação (ETZKOWITZ, 2003). Para ser empreendedora, a universidade assume considerável grau de independência do Estado e da indústria, mas também um alto nível de interação com essas esferas institucionais (ETZKOWITZ, 2013). A capacidade de tomar iniciativas independentes fundamenta-se na premissa de que a universidade não é um elemento subordinado de uma estrutura administrativa hierárquica (ETZKOWITZ, 2013). Umas das características importantes da universidade empreendedora é que a definição de problemas de pesquisa surge de fontes externas, a partir da interação entre os atores do desenvolvimento (ETZKOWITZ, 2003).

$\mathrm{Na}$ abordagem de sistemas nacionais de inovação, que envolve interações entre atores para promover o processo de inovação e de desenvolvimento regional, fica evidente o papel do governo como o principal ator responsável pelo estímulo financeiro e legal e por criar um ambiente propício à cooperação. Ou seja, o governo estimula a relação entre universidades e empresas por meio de recursos para projetos de pesquisa e para a formação de novas empresas, além de regulamentações que permitam a interação e a troca de conhecimento (LUNDVALL, 2007). Segundo o manual da OCDE 
(ORGANIZAÇÃO..., 2005, p. 31), “uma das principais tarefas dos governos é criar condições que induzam as empresas a realizarem os investimentos e as atividades inovadoras necessárias para promover a mudança técnica".

O governo é considerado um ator fundamental à medida que determina os incentivos e apoios para o desenvolvimento econômico, científico e tecnológico e fornece os recursos necessários para financiar pesquisa básica e incentivar e encorajar o empreendedor organizacional (ETZKOWITZ, 2003). O seu papel consiste em promover o contexto legal, atuar como gerador de demanda, criando novos mercados para gerar produtos de alta tecnologia, financiar ciência e tecnologia e investir em educação nas universidades (ETZKOWITZ et al., 2007), além de expandir as capacidades da universidade e da empresa, de modo que sejam capazes de cooperarem entre si. $\mathrm{Na}$ tríplice hélice o governo incentiva a pesquisa no interior das universidades e auxilia na transformação da pesquisa em atividade econômica. A empresa é um ator local de produção e o governo a base das relações contratuais que garantem as inter-relações entre atores da tríplice hélice (ETZKOWITZ, 2003, 2009).

Na tríplice hélice, o governo age como um empreendedor público, mantendo sua função reguladora, incentivando o surgimento de relações híbridas entre as esferas, tornando a inovação não mais exclusiva a uma única esfera, e sim o resultado entre suas interações. A interação agrega representantes das esferas, principalmente em âmbito regional, num processo de planejamento de baixo para cima, possibilitando criar e pôr em prática iniciativas políticas cooperativamente (ETZKOWITZ, 2003, 2009). Deve apoiar novos empreendimentos mediante mudanças no ambiente regulatório, incentivos fiscais e oferta de capital de risco público. Age como a fonte das relações contratuais que garantem interações estáveis e de câmbio entre as esferas institucionais (ETZKOWITZ; MELLO; ALMEIDA, 2005).

Em relação ao papel das empresas, quanto mais elas forem capazes de aprender por meio da interação com fontes externas, maior é o incentivo sobre as outras para seguirem o mesmo caminho. Isso aprimora a capacidade 
de inovar tanto de empresas individuais quanto dos sistemas econômicos aos quais elas pertencem (FAGERBERG, 2005). Conforme Dosi e Castaldi (2002), o futuro das empresas depende de diferentes combinações entre modos de organização e arranjos institucionais que afetam os processos de aprendizagem de indivíduos e organizações. A formação de uma empresa baseada no conhecimento, por intermédio da cooperação entre universidade e governo, torna-se uma questão central da estratégia de inovação, podendo resultar inclusive em um novo modo de produção em determinada região (ETZKOWITZ, 2009).

Na tríplice hélice, a indústria é o lócus da produção e principal usuário da inovação (ETZKOWITZ; MELLO; ALMEIDA, 2005). Tem assumido o papel da universidade no desenvolvimento e formação de pesquisa, muitas vezes, no mesmo nível da universidade (LUNDBERG, 2013).

\section{Incubadora}

A tríplice hélice torna-se uma plataforma para criação de novas instituições e formas organizacionais, resultado da cooperação entre as três esferas institucionais (ETZKOWITZ, 2003). Organizações híbridas surgem com o objetivo de mediar as interações entre universidade, indústria e governo (ETZKOWITZ; MELLO; ALMEIDA, 2005).

As incubadoras propiciam acesso a pesquisadores-chave (SOETANTO; JACK, 2013), o que pode gerar o surgimento de novas empresas de base tecnológica, muitas vezes resultantes de projetos de pesquisa e desenvolvimento (P\&D), que têm por objetivo permitir a transformação de ideias no meio acadêmico em atividade produtiva, materializada em produtos ou processos (CASSIOLATO et al., 1996). São reconhecidas como parte dos sistemas de inovação e se caracterizam por serem espaços planejados e preparados para receber empresas e pelo uso compartilhado da infraestrutura por um período determinado (LAHORGUE, 2004). 
Segundo Etzkowitz (2003), as incubadoras de empresas podem ser consideradas um dos melhores exemplos de organização híbrida e do funcionamento da tríplice hélice. Ela é tradicionalmente definida como uma estrutura de apoio à formação de empresas, um método de treinamento para que pessoas possam colaborar. Têm sido utilizadas para auxiliar a formação de empresas, para melhorar níveis tecnológicos de outras já existentes e criar empregos em regiões necessitadas (ETZKOWITZ, 2009).

Sua origem é creditada aos Estados Unidos, na região do Vale do Silício na década de 50, com o surgimento das primeiras incubadoras de empresas em parcerias com universidades e centros de pesquisas locais. A cooperação, resultado do esforço conjunto do governo, academias e indústrias, especialmente a partir da década de 70 , contribuiu significativamente para a proliferação de empresas de ponta nas áreas de computação, comunicação e eletrônica nos Estados Unidos (LAHORGUE, 2004).

O apoio da incubação sob a forma de rede e apoio às empresas possui um efeito positivo no desempenho das startups; o suporte à rede de relacionamentos modera a relação entre uma estratégia de exploração e o desempenho das startups, enquanto o apoio ao empreendedorismo modera a relação entre uma estratégia de crescimento de mercado e o desempenho das startups (SOETANTO; JACK, 2016).

No Brasil, o movimento de incubadoras surgiu em meados da década de 80, uma época marcada por uma grave crise econômica e pelo término do regime militar. O movimento ganhou força quando, principalmente após a democracia restaurada, os governos municipais incentivaram financeiramente as primeiras incubadoras e subsidiaram estrutura física para o seu funcionamento. Representou, assim, um modelo de desenvolvimento alternativo para a estratégia de "cima para baixo", incluindo associações industriais e universidades localizadas regionalmente a cooperar numa estratégia de 
"baixo para cima", criando um novo modelo de desenvolvimento menos custoso, aproveitando recursos acadêmicos, industriais e governamentais já existentes e facilitando a transferência de tecnologias entre universidades e indústrias (ETZKOWITZ, 2002, 2003).

De acordo com Terra et al. (2013), as incubadoras no Brasil executam atividades diretamente relacionadas ao crescimento tecnológico, econômico e educacional, incentivando o aporte de capital de risco como fonte necessária de investimento para o desenvolvimento de novos produtos e serviços.

O estudo realizado em incubadoras do Paraná por Pereira et al. (2014) demonstrou que os empreendedores percebem mais os benefícios tangíveis proporcionados pela incubadora, como o espaço físico, equipamentos e cursos ofertados. Constata-se, entretanto, que existem benefícios indiretos, como a contribuição para o reconhecimento de mercado e o apoio para intermediar relacionamentos benéficos à incubada, como o acesso a editais. Pode-se observar ainda que a intermediação da incubadora para desenvolvimento da inovação é secundária.

As incubadoras pesquisadas no Estado de Goiás proporcionaram benefícios limitados para a região em que estão instaladas, posto que existe um baixo número de projetos. No que tange às universidades, também foram poucos os benefícios diretos e os empreendedores relataram que nem todos os serviços são ofertados adequadamente, em especial aqueles de acesso ao capital financeiro (TONDOLO et al., 2016). Na pesquisa realizada em São Paulo por Storopoli et al. (2015), a infraestrutura tem uma importância maior para as empresas em estágio inicial de incubação em relação àquelas em estágio mais avançado de incubação, porém o networking foi apontado como um dos principais benefícios proporcionados pela incubadora.

Em um estudo realizado em oito incubadoras brasileiras de diversas regiões, os resultados revelam que não há prioridade para as empresas criadas a partir dos resultados de pesquisa acadêmica, apesar da preferência das incubadoras por projetos com alto potencial de interação com a universidade 
(STAL; ANDREASSI; FUJINO, 2016). Também existem poucos esforços para atrair o público acadêmico, o que leva à subutilização deste importante canal para a transferência de conhecimentos para a sociedade. A seguir serão apresentados os procedimentos metodológicos utilizados nesta pesquisa.

Neste artigo, entendemos a incubadora de empresas como um ambiente de inovação, que, segundo Zouain (2003), são ambientes que possuem a presença de instituições públicas e privadas que garantem condições favoráveis para o desenvolvimento de novos produtos e processos, minizando os riscos inerentes às iniciativas de natureza inovadoras e maximizando os seus resultados.

\section{Procedimentos Metodológicos}

A pesquisa caracterizou-se como um estudo de caso exploratório e descritivo. Conforme Yin (2005), estudo de caso é uma estratégia apropriada quando aplicada sobre um conjunto de acontecimentos que não é controlado pelo pesquisador, desenvolvido em um território delimitado, nesse caso, na Incubadora Tecnológica de Luzerna, localizada no meio- oeste catarinense. $\mathrm{O}$ artigo assumiu uma abordagem qualitativa que, conforme Godoy (1995), oferece subsídios para a interpretação e compreensão dos processos que envolvem a administração, sobretudo quando busca compreender os fenômenos segundo a perspectiva do sujeito, ou seja, dos participantes da situação em estudo.

A seleção dos artigos para a elaboração da fundamentação teórica foi realizada mediante pesquisa bibliográfica utilizando a base de dados Scopus, reconhecida como uma das maiores fontes referenciais de literatura técnica e científica revisada por pares (ELSEVIER, 2014). Por meio da busca pelas palavras-chave "triple helix" e "business incubador", foram selecionados os 50 artigos mais citados de cada tema. A escrita do referencial teórico não se limitou apenas aos cem artigos, foram utilizados também livros, dissertações, teses e demais artigos relativos ao tema. 
Após a escolha dos artigos, procedeu-se à leitura e à proposição preliminar de categorias de análise relacionadas aos fatores que determinam a constituição de ambientes de inovação. O processo foi realizado com auxílio do software Nvivo versão 10. Conforme Mozzato e Grzybovski (2011), é um software de dados qualitativos que tem por objetivo facilitar, agilizar, validar e gerar confiança, qualificando o material coletado.

Como o método de estudo de caso exige cuidados especiais nos procedimentos de coleta de dados e deve contar com múltiplas fontes de evidência para garantir a qualidade de informações obtidas de forma triangular (YIN, 2005), foram utilizadas análise documental, observação não participante e entrevistas.

Para análise documental foram utilizados registros impressos e digitais da ITL, editais de fomento à inovação, legislações, matérias de jornais e revistas, entre outros. A observação não participante foi executada com auxílio de um protocolo de anotações de campo de natureza reflexiva e que possibilitou corrigir falhas no decorrer da pesquisa e registrar expressões e ações realizadas pelos sujeitos de pesquisa. Em relação à entrevista, foi efetivada com o auxílio de um roteiro semiestruturado que apresentou questões abrangentes em seu enunciado principal, do tipo "como", "por que", "quem" e "quando" foi constituída a ITL, e questões adicionais em forma de tópicos, que foram introduzidas, quando necessário, de forma natural, à medida que se estabelecia o diálogo entre o entrevistado e o pesquisador. $\mathrm{O}$ mesmo roteiro de entrevista foi aplicado aos representantes da universidade, governo, empresa e da ITL.

A escolha dos entrevistados em uma pesquisa qualitativa, segundo Alberti (2013), deve priorizar aqueles que participaram ou testemunharam ocorrências ou situações ligadas ao tema e que possam fornecer depoimentos significativos. A entrevista foi realizada com representantes das esferas institucionais da tríplice hélice e que tiveram participação direta e efetiva na constituição da ITL. Ou seja, o critério utilizado para escolha dos entrevistados respeitou: a participação dos atores que contribuíram para a 
constituição da ITL e os sujeitos que participaram efetivamente do processo de instalação e manutenção do ambiente de inovação. As entrevistas foram gravadas e posteriormente transcritas. No Quadro 1 é possível visualizar a relação dos entrevistados, instituição que representa, local de realização da entrevista e tempo de duração:

\section{Quadro 1 - Relação dos entrevistados}

\begin{tabular}{|c|c|c|c|}
\hline Entrevistado & Ator & Local & Duração \\
\hline Entrevistado A & Governo & Prefeitura de Luzerna & $58 \mathrm{~min}$ \\
\hline Entrevistado B & \multirow[t]{2}{*}{ Universidade } & $\begin{array}{c}\text { Universidade do Oeste de Santa Catarina } \\
\text { - Unoesc Joaçaba }\end{array}$ & $76 \mathrm{~min}$ \\
\hline Entrevistado $\mathrm{C}$ & & $\begin{array}{c}\text { Instituto Federal Catarinense - Campus } \\
\text { de Luzerna }\end{array}$ & $34 \mathrm{~min}$ \\
\hline Entrevistado D & Empresa & Incubadora Tecnológica de Luzerna & $42 \mathrm{~min}$ \\
\hline Entrevistado $\mathrm{E}$ & $\begin{array}{l}\text { Organização } \\
\text { híbrida }\end{array}$ & Incubadora Tecnológica de Luzerna & $31 \mathrm{~min}$ \\
\hline Entrevistado F & Empresa & Realizado via skype & $15 \mathrm{~min}$ \\
\hline Entrevistado $G$ & $\begin{array}{l}\text { Organização } \\
\text { híbrida }\end{array}$ & Realizado via skype & $16 \mathrm{~min}$ \\
\hline
\end{tabular}

Fonte: Elaborado pelos autores.

Em relação à quantidade de entrevistados necessária para o desenvolvimento do estudo houve a preocupação de realizar entrevistas capazes o suficiente de fornecer as informações indispensáveis, ou seja, a qualidade das informações prestadas tornou-se prioridade diante da quantidade de entrevistas previamente estabelecidas. Conforme Alberti (2013), cabe ao pesquisador avaliar se o grau de informações obtidas é suficiente para construir uma interpretação bem fundamentada sobre o tema de estudo.

Para estudo dos dados optou-se pela análise de conteúdo (BARDIN, 2009) e a utilização de categorias de grade aberta, categorias que surgem durante o tratamento de dados e sofrem alterações até se chegar às categorias finais de análise (VERGARA, 2010), ou seja, o estudo bibliográfico forneceu 
categorias preliminares de análise que sofreram ajustes no tratamento de dados sobre as fontes de evidência, em especial as entrevistas. A análise de conteúdo foi realizada com auxílio do software QSR NVivo versão10.

\section{Análise dos Dados e Discussão}

A Incubadora Tecnológica de Luzerna (ITL) foi instituída pela Lei n. 866, de 27 de outubro de 2009 e inaugurada em agosto de 2010. Uma iniciativa do poder público municipal, com apoio da Fundação de Amparo à Pesquisa e à Inovação do Estado de Santa Catarina (Fapesc). É considerada a única incubadora pública do Estado de Santa Catarina, mantida pela prefeitura de Luzerna, uma cidade localizada no meio-oeste catarinense, com aproximadamente 6 mil habitantes, que se destaca no ramo metalmecânico e elétrico pela presença de empresas de pequeno e médio portes. Instalada em um antigo seminário franciscano, ocupa uma área de $380 \mathrm{~m}^{2}$, possui atualmente dez empreendimentos instalados nas áreas de metalmecânica, eletrônica, automação, agroindústria, engenharia biomédica, alimentos e energias renováveis. Além da infraestrutura, como sala, telefone, Internet, sala de recepção, sala de reuniões, auditório com capacidade para 130 lugares, laboratórios e oficina, oferece o serviço de apoio ao incubado realizado pela própria secretaria da ITL (INCUBADORA..., 2014).

A ITL é classificada como uma incubadora mista que, conforme Lahorgue (2004), abriga empresas de base tecnológica, cujos produtos, processos ou serviços são resultados de pesquisa científica, representando um alto valor agregado por intermédio da tecnologia; e abriga empresas de setores tradicionais, ou seja, empreendimentos ligados a áreas da economia que detêm tecnologias difundidas e desejam agregar valor a seus produtos.

A estrutura organizacional da ITL é composta por um Conselho de Desenvolvimento Econômico e Tecnológico, constituído por representantes da: Secretaria Municipal da Fazenda e Administração de Luzerna (SMFAL); Associação dos Comerciantes, Industriais e Artesãos de Luzerna (Acial); 
gestor da ITL; representantes das empresas incubadas; Assessoria de Indústria e Comércio (AIC); Conselho do município indicado pelos conselheiros; Instituto Federal Catarinense (IFC); Serviço Nacional de Aprendizagem Industrial (Senai); Universidade do Oeste de Santa Catarina (Unoesc), Serviço Brasileiro de Apoio às Micro e Pequenas Empresas (Sebrae) e comunidade externa. Também é composta por um gestor responsável pela administração geral da ITL, nomeado pelo prefeito de Luzerna e por um Conselho Consultivo das Empresas Incubadas, formado por um representante de cada empresa.

\section{Fatores que Determinaram a Constituição da Incubadora Tecnológica de Luzerna à luz da Tríplice Hélice}

A partir da análise de conteúdo foi possível identificar os principais fatores que determinaram a constituição da ITL. Os fatores foram categorizados com o auxílio do software Nvivo, os quais são: a existência de lideranças, iniciativa do poder público municipal, oportunidade e continuidade do trabalho desenvolvimento pela pré-incubadora da universidade, presença de instituições públicas e privadas, inexistência de outras incubadoras em municípios próximos e a necessidade de criar uma nova matriz econômica para a região.

Os fatores determinantes identificados como responsáveis pela constituição da ITL corroboram com a crítica de Viale e Campodall'orto (2002) ao modelo tríplice hélice, que desempenha, muitas vezes, segundo os autores, uma função meramente prescritiva e de generalização empírica de sistemas locais de inovação, e segundo Leydesdorff (2012), estudos empíricos são necessários para explorar o modelo. $\mathrm{O}$ fator pessoas, ou seja, as lideranças envolvidas no processo de constituição da ITL foram determinantes na efetivação da incubadora e foi mencionado pelos entrevistados como o principal. Recebe destaque a iniciativa do Entrevistado A em buscar recursos na Fapesc, por incentivar as instituições públicas e 
privadas da região para cooperarem em prol de um ambiente de inovação e por acreditar que inovação, via interação entre universidade, governo e empresas, é capaz de promover novas demandas econômicas e gerar empregos qualificados. Ademais, ainda destaca-se o papel do Entrevistado D e atual diretor da empresa âncora do empreendimento, pela postura proativa em buscar alternativas para a gestão e sustentabilidade da ITL, por propor melhorias no processo de incubação das empresas, incentivar a colaboração interna e externa das empresas incubadas, e por acreditar e defender que o ambiente de inovação pode se tornar uma nova alternativa econômica, social e tecnológica para a região.

O fator pessoas mostra-se importante na constituição da ITL por ser um determinante responsável em criar iniciativas em prol do desenvolvimento da região a partir de um modelo de inovação, e ser responsável pela execução das ações necessárias para tal, criando um ambiente de cooperação entre diferentes atores que até então interagiam de maneira modesta e isolada.

Figura 1 -Fatores determinantes na constituição da ITL à luz da tríplice hélice

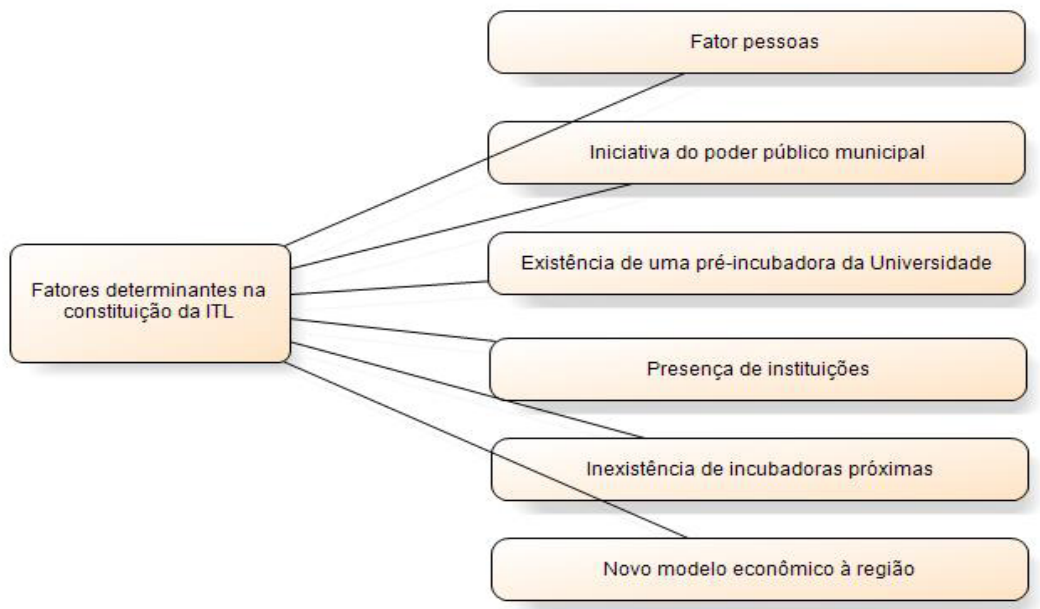

Fonte: Elaborada pelos autores no Software Nvivo 
Além de serem responsáveis pela efetiva constituição da ITL, tanto o Entrevistado A quanto o Entrevistado D participaram efetivamente das atividades de pré-incubação na universidade como professor e aluno, participando de edições do Sinapse de Inovação, uma iniciativa do governo do Estado de Santa Catarina, cujo objetivo é transformar e aplicar ideias geradas por estudantes, pesquisadores e profissionais. A participação no Sinapse de Inovação na pré-incubadora da universidade, conforme o Entrevistado A, possibilitou conhecer iniciativas e modelos de outras cidades do Estado e encorajou a criação de uma incubadora no meio-oeste catarinense.

Nesse contexto, a existência de uma pré-incubadora da universidade contribui significativamente para a constituição da ITL. As duas primeiras empresas a se instalarem na ITL passaram por um período de pré-incubação de aproximadamente dois anos na universidade. Pode-se afirmar que a pré-incubação da universidade foi umas das primeiras iniciativas organizadas a envolver os atores da tríplice hélice para apoiar e incentivar a inovação na região, mas a ITL teve um papel de destaque, por envolver as instituições em um caráter formal de cooperação.

A existência de uma pré-incubadora foi importante na constituição da ITL, na visão do representante da universidade, por incentivar a criação de novos negócios e possibilitar a continuidade da criação de uma incubadora, suprindo a necessidade de algumas empresas em desenvolverem seus produtos e processos em um ambiente propício e adequado. Ainda, segundo o Entrevistado B, a ITL pode ser considerada, atualmente, um expoente do incentivo à interação com os atores da tríplice hélice da região.

A iniciativa do poder público municipal em participar de editais de recursos da Fapesc, para a criação da incubadora no Estado em 2009 foi fundamental. Conforme o Entrevistado A, existia na época a vontade do poder público em disponibilizar infraestrutura para alocar empresas de base tecnológica para constituir seus negócios, mas a preocupação de fato não era a necessidade de recursos para a construção de novos prédios, e sim constituir um espaço, um ambiente em que empresas pudessem cooperar e 
ser auxiliadas por outras instituições por meio dos seus papéis tradicionais. Com os recursos da Fapesc, foi possível reformar um antigo seminário e contratar consultores para dar início às atividades da ITL.

A colaboração dos atores por meio de seus papéis tradicionais é considerada por Etzkowitz (2003, 2009) e Etzkowitz et al. (2008) como o primeiro passo em direção à tríplice hélice. Nesse sentido, o poder público municipal reconheceu a necessidade de envolver várias instituições públicas, privadas e a sociedade civil organizada para participar do Conselho de Desenvolvimento Econômico e Tecnológico da ITL e se envolver nas atividades de apoio e incentivo à inovação.

A necessidade de novos modelos de desenvolvimento econômico para a região e a preocupação da comunidade também foram apontadas como fatores que influenciaram a origem da incubadora e das empresas de base tecnológica em uma região, cuja principal atividade está na agricultura. De acordo com o Entrevistado E, a incubadora está incentivando uma nova forma de desenvolvimento e uma esperança para as pessoas que residem na região, a de que elas podem estudar e constituir os seus negócios, não mais necessitando mudar para grandes centros. Nas palavras do Entrevistado E:

O aluno que está estudando aqui, que tinha a percepção de ir embora, agora ele pensa, "puxa eu não preciso ir mais embora". A sua maior contribuição da ITL é mudar o atual contexto, a mentalidade das pessoas, o ambiente e dizer, "puxa, nós também podemos desenvolver a nossa região", podemos ter empresas de base tecnológica, ou seja, pegar o empreendedor e dar um amparo para ele, essa é a maior contribuição. Nós estamos vislumbrando um bom futuro para a nossa região.

Umas das características marcantes do modelo da tríplice hélice refere-se aos recursos necessários à formação e operacionalização da tríplice hélice, fornecidos a partir das condições locais/regionais. Infraestrutura existente, 
características regionais e institucionais dos atores envolvidos são elementos fundamentais de constituição da tríplice hélice (ETZKOWITZ, 1997, 2003, 2009; ETZKOWITZ et al., 2008; LEYDESDORFF; ETZKOWITZ, 2003).

A preocupação em criar novos modelos e matrizes econômicas é considerada um determinante na constituição da ITL. Sua importância é fortalecida à medida que as lideranças locais discutem sobre possíveis alternativas de diversificação da matriz produtiva regional diante da competitividade global, e um olhar sobre profissões futuras e novos empreendimentos baseados no conhecimento, passíveis de serem criados na região de abrangência da incubadora.

A existência de instituições públicas e privadas na região também contribuiu para a constituição da ITL. Recebe destaque a presença da Universidade do Oeste de Santa Catarina (Unoesc) na formação de lideranças, no desenvolvimento regional e por criar iniciativas de incentivo e apoio à inovação, como é o caso da pré-incubadora e do Polo de Inovação do Rio do Peixe (Inovale).

Outro fator identificado é a inexistência de incubadoras próximas. A ITL abrange, aproximadamente, 14 municípios do meio-oeste catarinense, que concentram no município de Luzerna suas atividades de incubação. Conforme Zouain (2005), disputas entre regiões podem enfraquecer projetos de ambientes de inovação e prejudicar a articulação de políticas públicas para o desenvolvimento regional. Nesse contexto, pode-se aferir que, ao constituir um ambiente de inovação, é necessário explorar o potencial de capacidade de demanda e oferta de tecnologia da região. Ações de ambientes isolados correm o risco que fracassarem antes mesmo de se constituírem.

\section{Papel da Universidade, Governo e Empresas Assumido na Constituição da ITL}

A interação entre universidade, governo e indústria para promover o desenvolvimento regional e a inovação é um dos pontos-chave do modelo da tríplice hélice. Cada esfera institucional, além do seu papel tradicional, 
envolve-se em outras atividades que visam a promover a inovação por meio da interação em ambientes de inovação. Buscar compreender o papel assumido pelas esferas institucionais é importante à medida que fornece elementos que possam servir de base para outros ambientes e contextos, como, também, estudar a viabilidade do modelo tríplice hélice em regiões que careçam de novas alternativas de desenvolvimento. A seguir serão apresentados os papéis assumidos pelas esferas institucionais em um ambiente de inovação, neste caso, o da ITL.

A contribuição do governo, quer seja por buscar recursos financeiros, por disponibilizar o local de instalação ou por unir diferentes entidades e instituições, assume destaque diante das demais esferas institucionais na constituição da ITL. No papel assumido por parte da universidade destacam-se a existência de uma pré-incubadora, a formação de profissionais e a geração do conhecimento. No papel das empresas, por sua vez, ressalta-se a colaboração interna entre os incubados e o papel da empresa âncora no fortalecimento da imagem da ITL. As categorias de análise do papel da universidade, governo e empresa podem ser visualizadas na Figura 2.

Figura 2 - Papéis da universidade, governo e empresa assumidos na ITL

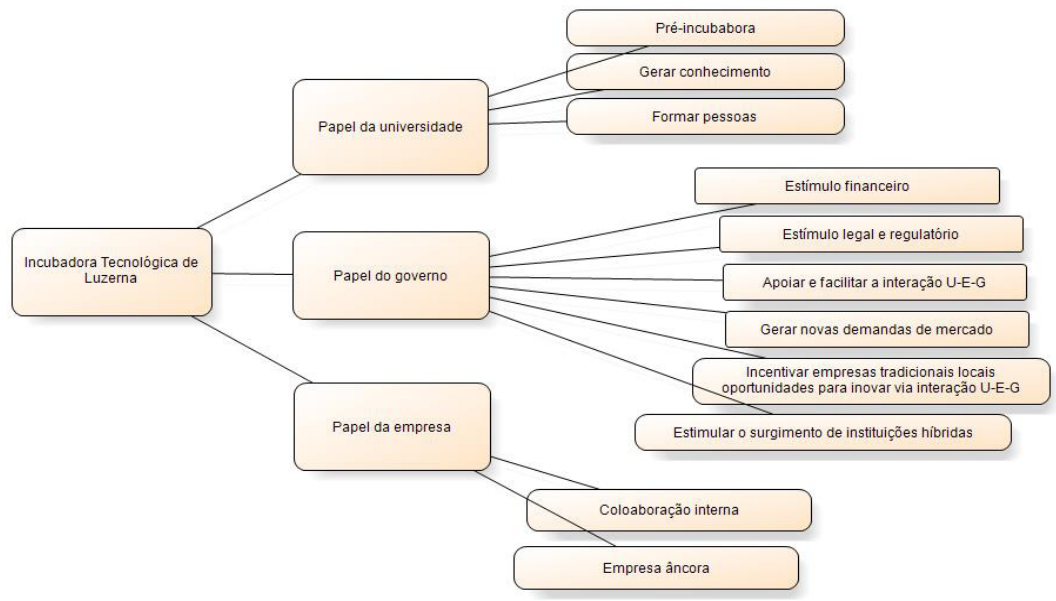

Fonte: Elaborado pelos autores no Software Nvivo 
Em relação ao papel do governo para a constituição da ITL, o estímulo financeiro inicial promovido pela iniciativa do poder público municipal em captar recursos via Fapesc, do governo estadual, foi determinante para a instalação da ITL, principalmente em relação à infraestrutura necessária para o início das atividades. O papel do governo quanto ao estímulo financeiro para a ITL corrobora com os estudos de Etzkowitz (2003), Etzkowitz, Mello e Almeida (2005) e Lundvall (2007).

O estímulo legal e regulatório às empresas também pode ser observado na lei de inovação do município de Luzerna, ou seja, a Lei n. 615, de 20 de outubro de 2005, a qual dispõe sobre a política municipal de desenvolvimento econômico, incentivos econômicos e fiscais para empresas que se estabelecerem, ampliarem sua capacidade produtiva ou desenvolverem projetos de desenvolvimento tecnológico e inovação. $\mathrm{O}$ incentivo ocorre mediante concessão do direito de uso de área de terra, facilidade de acesso ao local de instalação da empresa, coparticipação na rede de água, telefone, energia elétrica, isenção de impostos e taxas municipais, concessão de espaço físico para empresas incubadas, compartilhamento de laboratórios, equipamentos, instrumentos, materiais e demais instalações. O papel do governo quanto ao estímulo legal e regulatório é mencionado por Etzkowitz (2003, 2009), Etzkowitz, Mello e Almeida (2005) e Lundvall (2007).

A geração de novas demandas de mercado, mencionadas por Etzkowitz et al. (2007) como um papel do governo, também pode ser observada no caso da ITL. O Entrevistado A relata sua preocupação com o futuro do município, que depende atualmente, quase que exclusivamente, da receita de matrizes econômicas tradicionais da região. Incentivar o surgimento de empresas de base tecnológica pode ser uma alternativa para o município prosperar em termos de crescimento econômico e desenvolvimento. Nas palavras do Entrevistado A:

Para o governo, é uma nova forma, e eu diria, de trabalhar a sustentabilidade, de não ficar na mão somente de empresas tradicionais, ou seja, o nosso município depende da receita das grandes empresas e da agricultura, aproximadamente $40 \%$ hoje da receita é da agricultura, mas como vai ser daqui 5 ou 10 anos? 
Ao contribuir com a constituição da ITL, o governo passa a apoiar e facilitar as interações entre universidade, empresa e governo, antes despercebidas no município. Esse papel corrobora com Etzkowitz, Mello e Almeida (2005), Etzkowitz (2003, 2009) e Lundvall (2007).

Incentivar empresas locais para inovar via interação entre universidade, empresa e governo foi uma categoria que emergiu dos estudos e que apresenta um novo desafio ao governo municipal. A existência da ITL cria oportunidades de as empresas locais interagirem com o ambiente de inovação, aprimorando seus produtos e processos, mas que nem sempre é aproveitada pelas empresas locais. O Entrevistado A destaca que:

O grande desafio nosso hoje é justamente isso, mostrar às empresas tradicionais as oportunidades de formalizar e profissionalizar o aprimoramento de seus novos produtos.[...] Olha, tem alunos pesquisando o tempo todo e necessitando de novas coisas para estudar, por que não amarrar o desenvolvimento de produto da empresa com a universidade e com o poder público apoiando?

Corroborando com essa preocupação, o Entrevistado D menciona que:

Estamos correndo atrás disso, mas só vamos conseguir se todos pensarem dessa forma e plantar essa concepção que podemos crescer e desenvolver colaborando uns com os outros. As pessoas que estão vendo isso pensam, "puxa, como é possível ter isso aqui".

Ao serem questionados quanto às barreiras e dificuldades do governo em interagir com a universidade e empresas no contexto da ITL, a morosidade e burocracia dos processos prevaleceu nas respostas dos entrevistados. Nas palavras do Entrevistado F: "Vejo que as dificuldades impostas pela máquina pública deixam morosos os trâmites de uma simples troca de fechadura, afinal temos que partir do princípio de legalidade" 
$\mathrm{Na}$ visão do Entrevistado D, as empresas incubadas e o próprio Entrevistado E já assumem uma posição proativa na aquisição de materiais para o funcionamento da incubadora e na preparação de salas para acomodação de novas empresas. Nas palavras do Entrevistado E: "Hoje as taxas das empresas são revertidas, $90 \%$ para o conselho da ITL e $10 \%$ para a prefeitura, assim conseguimos bancar algumas coisas que são urgentes aqui dentro”. Ser autossuficiente é um desafio e necessidade da ITL, destaca o Entrevistado E. A preocupação também é enfatizada pelo Entrevistado C, quando menciona que uma das "desvantagens da gestão da ITL ser pública é a troca de governos a cada quatro anos e que pode quebrar todo o trabalho que vem sendo feito anteriormente, o que não pode acontecer com a inovação, pois a gente sabe que inovação dá retorno a longo prazo".

Ao serem questionados quanto às barreiras e dificuldades do ator empresa, chamou a atenção o fato de as empresas atuantes e tradicionais da região não terem conhecimento da ITL o do seu papel no auxílio para o desenvolvimento de inovações. Além de não conhecerem o ambiente criado para a promoção da inovação, não utilizam procedimentos de cooperação com o governo e a universidade. Nas palavras do Entrevistado G: "A principal dificuldade é conseguir a credibilidade para possíveis parcerias, pois no setor tradicional no município de Luzerna os empresários desconhecem o conceito de startup e incubadora de empresas, o que dificulta uma aproximação e possíveis parcerias”. O Entrevistado A alerta que, muitas vezes, produtos e serviços poderiam ser desenvolvidos em ambientes de colaboração recíproca, profissionalizando o desenvolvimento do produto e potencializando-o para o mercado, ou seja, todos poderiam ganhar com isso.

Por sua vez, a ITL destaca-se pelo ambiente colaborativo interno das empresas incubadas, conforme palavras do Entrevistado E:

O que acontece aqui, como todas as empresas estão no corredor, acabamos involuntariamente tratando de negócios e de projetos, e um ajuda o outro. Eu tenho conhecimento de uma coisa que os outros não têm. E 
se nós não conversamos com outras empresas? De onde vamos tirar isso? A informação é de graça aqui dentro, as pessoas estão uma do lado das outras e se ajudam. No ambiente de incubação, como as empresas estão todas próximas, todo mundo no mesmo barco, é mais fácil. Lá fora não existe esse contato informal e colaborativo. Se uma empresa não tem o conhecimento para construir uma máquina, a outra empresa ajuda, ou seja, acabam negociando entre as empresas da ITL criando um ambiente propício para a inovação e o desenvolvimento, e acaba que a empresa maior puxa as outras e se fortalecem.

A empresa âncora recebe destaque por incentivar as demais para inovação e, também, por prestar auxílio para aquelas que estão iniciando e, em algumas vezes, prestando o suporte financeiro necessário às demais. O fato de a empresa âncora iniciar o seu empreendimento na ITL, por ter passado por experiências de desenvolver novos produtos, interagir com o governo e a universidade e depositar seus pedidos de patentes, é vista pelas demais como um exemplo a ser seguido, ou seja, a empresa âncora, no caso da ITL, caracteriza-se por ser colaborativa, por legitimar o processo de incubação como uma alternativa para demais empreendedores investirem e apostarem em empresas de base tecnológica em uma região que apresenta características de economia agrícola e empresas do setor metalmecânico.

Ao serem questionados quanto às barreiras e dificuldades do ator universidade, destaca-se a morosidade de processos burocráticos, o que, muitas vezes, interfere na interação dos atores e a incerteza sobre a continuidade de programas de incentivo à inovação e interação.

\section{Conclusões}

A partir da pesquisa sobre os fatores que determinaram a constituição da ITL, foi possível identificar as contribuições que o modelo da tríplice hélice pode proporcionar para o desenvolvimento regional, aliado à iniciativa 
de lideranças dispostas a transformar uma realidade, criar novas alternativas de desenvolvimento econômico e tecnológico, incentivar a cultura de cooperação e interação entre diferentes atores para inovar.

No decorrer de apenas quatro anos de atividades da ITL, alguns resultados podem ser visualizados: oportunidades de novas alternativas de desenvolvimento econômico e tecnológico para a região; criação de empresas de base tecnológica em uma região cuja principal atividade é agrícola; aproximação da universidade, governo e empresas para participar de editais de fomento à inovação e captação de recursos; retenção de jovens talentos e mão de obra qualificada; incentivo para a realização de seminários e eventos para discutir e fomentar a interação entre atores para a inovação; tentativas de internacionalização da ITL via convênio firmado com a Irlanda, visando a intercâmbios culturais e tecnológicos; depósito de dez pedidos de patentes na área da saúde, Odontologia e Educação Física, entre outros; geração de novas tecnologias que facilmente podem ser absorvidas por outras empresas da região; internacionalização da empresa âncora, o que ampliou a visão de mercado das demais empresas incubadas e a criação de empresas de serviços especializados.

Dos principais fatores identificados na constituição da ITL à luz da tríplice hélice, destacam-se: (1) fator pessoas, ou seja, lideranças representantes da universidade, governo e empresa diretamente envolvidas e dispostas a criar um ambiente alternativo de aprendizagem e de geração de novas tecnologias e demandas de mercado; (2) a existência da pré-incubadora da universidade como um elemento antecedente, um ambiente propício ao aprendizado, criando as primeiras iniciativas de incentivo à inovação via interação entre universidade, governo e empresa da região, e (3) contribuição do poder público municipal mediante incentivos financeiros, fiscais e regulatórios, e pela iniciativa de unir diferentes setores da sociedade para apoiar e incentivar a inovação via um ambiente de inovação, como a ITL. 
Em relação ao papel dos atores, pôde-se observar um movimento de interação recíproco para o surgimento da ITL, cada qual a partir de seus papéis tradicionais, seguindo assim as prerrogativas do modelo tríplice hélice. $\mathrm{Na}$ constituição da ITL a esfera institucional do governo assume posição de destaque pela iniciativa de fomentar recursos para construção (reforma) de uma infraestrutura de acolhimento da ITL, bem como gerar expectativas para o empreendedorismo e a inovação para a região. À medida que o tempo passa e os processos exigem amadurecimento e transformação por partes das instituições, a dimensão empresa e a gestão da ITL assumem destaque por criar mecanismos de gestão, cooperação entre as empresas incubadas e formas de sustentabilidade do ambiente de inovação.

Para estudos futuros propõe-se estudar os fatores determinantes na constituição de outros ambientes de inovação, geridos pelo poder público municipal, como também por universidades e empresas, possibilitando comparações entre as diferentes formas híbridas que surgem e os diferentes papéis assumidos pelas esferas do governo, universidade e indústria. Também sugere-se pesquisas futuras capazes de acompanhar os resultados da inovação gerados pela ITL, além de pesquisas capazes de explorar elementos que dificultam e/ou facilitam o desempenho das incubadoras para atingir seus objetivos de gerar e difundir a inovação.

\section{Referências}

AUDY, J. L. N. Ambiente da inovação brasileira. Revista Locus. Anprotec, ano 20, n. 77, set. 2014. Disponível em: <http://anprotec.org.br/site/revista-locus/>. Acesso em: 27 jan. 2015.

ALBERTI, V. Manual de história oral. 3. ed. Rio de Janeiro: Ed. FGV, 2013.

BARDIN, L. Análise de Conteúdo. Ed. rev. e atual. Lisboa: Edições 70, 2009.

CASSIOLATO, J. E. et al. A relação universidade e instituições de pesquisa com o setor industrial: uma análise de seus condicionantes. Rio de Janeiro: IE; UFRJ, 1996. 
DOSI, G.; CASTALDI, C. Padrões locais e divergentes de aprendizagem tecnológica em mercados (parcialmente) globalizados. In: CASTRO, A. C. (Org.). Desenvolvimento em Debate: Novos rumos do desenvolvimento no mundo. Rio de Janeiro: BNDES, 2002.

ELSEVIER. Scopus. 2014. Disponível em: <http:/www.americalatina.elsevier.com/ corporate/scopus.php>. Acesso em: 15 dez. 2014.

ETZKOWITZ, H. Anatomy of the entrepreneurial university. Social Science Information, v. 52, n. 3, p. 486-511, 2013.

. Hélice Tríplice: Universidade-indústria-governo: inovação em ação. Porto Alegre: EDIPUCRS, 2009.

. Innovation in innovation: the triple helix of university-industry- government relations. Social Science Information, v. 42, n. 3, p. 293-337, 2003.

Networks of Innovation: Science, Technology and Development in the Triple Helix Era. International Journal of Technology Management and Sustainable Development, v. 1-1, p. 7-20, 2002.

. The entrepreneurial university and the emergence of democratic corporatism. Universities and the global knowledge economy: a triple helix of university-industrygovernment relations. London: Pinter, p. 141-152, 1997.

ETZKOWITZ, H. et al. Pathways to the entrepreneurial university: towards a global convergence. Science and Public Policy, v. 35, n. 9, p. 681-695, 2008.

. The Creation of Born Global Companies within the Science Cities: An approach from Triple Helix. Engevista, v. 9, n. 2, p. 149-164, 2007.

ETZKOWITZ, H.; KLOFSTEN, M. The innovation region: toward a theory of knowledge - based regional development. R\&DD Management 35, MA, USA: Blackwell Publishing Ltd., 2005.

ETZKOWITZ, H.; LEYDESDORFF, L. The Triple Helix - University, Industry, Government Relations: A Laboratory for Knowledge Based Economic Development. In: The Triple Helix of University, Industry, and Government Relations: the Future Location of Research Conference. Amsterdam, 1996.

ETZKOWITZ, H.; MELLO, J. M. C.; ALMEIDA, M. Towards “meta innovation” in Brazil: The evolution of the incubator and the emergence of a triple helix. Research Policy, n. 34, p. 411-424, 2005.

FAGERBERG, J. Innovation: A guide to the literature. In: FAGERBERG, J.; MOWERY, D. C.; NELSON, R. R. (Ed.). The Oxford Handbook of Innovation. New York: Oxford University Press Inc., 2005.

FAURÉ, Y.; HASENCLEVER, L. (Org.). Caleidoscópio do desenvolvimento local no Brasil. Ed. E-papers, Rio de Janeiro, 2007.

GODOY, A. S. Introdução à pesquisa qualitativa e suas possibilidades. Revista de Administração de Empresas, São Paulo, v. 35, n.2, p.57-63, 1995. 
HID, D. S.; NASCIMENTO, C.; OLIVEIRA, D. A. Análise das publicações internacionais relacionadas ao desenvolvimento sustentável na área de administração: uma análise bibliométrica da produção científica. Administração: ensino e pesquisa, Rio de Janeiro, v. 13 n. 4, p. 653-671, out./dez. 2012.

INCUBADORA TECNOLÓGICA DE LUZERNA. Disponível em:<http://www. incubadoraluzerna.com.br>. Acesso em: 15 dez. 2014.

JOHNSON, B.; EDQUIST, C.; LUNDVALL, B. Economic development and the national system of innovation approach. In: FIRST GLOBELICS CONFERENCE. 1, 2003, Rio de Janeiro. Anais... Rio de Janeiro: Redesist, 2003, p. 1-24.

LAHORGUE, M. A. Pólos, parques e incubadoras: instrumentos de desenvolvimento do século XXI. Anprotec, 2004.

LEYDESDORFF, L. The triple helix, quadruple helix, and an N-tuple of helices: Explanatory models for analyzing the knowledge-based economy? Journal of the Knowledge Economy, v. 3, n. 1, p. 25-35, 2012.

. The triple helix model and the study of knowledge based innovation systems. International Journal of Contemporary Sociology, 2005.

LEYDESDORFF, L.; ETZKOWITZ, H. Can "the public" be considered as a fourth helix in university-industry-government relations? Report on the Fourth Triple Helix Conference, 2002. Science and Public Policy, v. 30, n. 1, p. 55-61, 2003.

. The triple helix as a model for innovation studies. Science and public policy, v. 25, n. 3, p. 195-203, 1998.

LUNDBERG, H. Triple Helix in practice: the key role of boundary spanners. $E u$ ropean Journal of Innovation Management, v. 16, n. 2, p. 211-226, 2013.

LUNDVALL, B. A. National innovation systems - analytical concept and development tool, Conference Dynamics of Industry and Innovation: Organizations, Networks and Systems, 27-29 june, Copenhagen. 2007.

MEYER, M.; SINILÄINEN, T.; UTECHT, J.T. Towards hybrid Triple Helix indicators: A study of university-related patents and a survey of academic inventors. Scientometrics, v. 58, n. 2, p. 321-350, 2003.

MOZZATO, A. R.; GRZYBOVSKI, D. Análise de conteúdo como técnica de análise de dados qualitativos no campo da administração: potencial e desafios. Revista de Administração Contemporânea, Curitiba, v. 15, n. 4, p. 731-747, jul./ago. 2011.

ORGANIZAÇÃO PARA A COOPERAÇÃO E DESENVOLVIMENTO ECONÔMICO (OCDE). Manual de Oslo: diretrizes para coleta e interpretação de dados sobre inovação. 3. ed. Brasília: Finep, 2005.

PEREIRA, J. A. et al. A incubadora como intermediária de relacionamentos na percepção dos empreendedores: um estudo multicaso em incubadoras paranaenses. Gestão e Sociedade, v. 8, n. 19, p. 496-516, 2014. 
SAAD, M.; ZAWDIE, G.; MALAIRAJA, C. The triple helix strategy for universities in developing countries: the experiences in Malaysia and Algeria. Science and Public policy, v. 35, n. 6, p. 431-443, 2008.

SCHOONMAKER, M. G.; CARAYANNIS, E. G. Mode 3: a proposed classification scheme for the knowledge economy and society. Journal of the Knowledge Economy, v. 4, n. 4, p. 556-577, 2013.

SOETANTO, D.; JACK, S. The impact of university-based incubation support on the innovation strategy of academic spin-offs. Technovation, v. 50-51, p. 25-40, 2016.

. Business incubators and the networks of technology-based firms. Journal of Technology Transfer, v. 38, p. 432-453, 2013.

STAL, E.; ANDREASSI, T.; FUJINO, A. the Role of University Incubators in Stimulating Academic Entrepreneurship. RAI: Revista de Administração e Inovação, v. 13, n. 2, p. 27-47, 2016.

STOROPOLI, J. E. et al. O desenvolvimento de capacidades e recursos em incubadoras de empresas. Revista Ciências Administrativas, v. 21, n. 1, p. 1-15, 2015.

TERRA, B. et al. Interaction Among Universities, Government and Spin-off Companies in a Brazilian Context to Generate Sports Innovation. Journal of technology management छ innovation, v. 8, n. 2, p. 93-106, 2013.

TONDOLO, L. P. et al. Incubadoras de empresas: muitos investimentos públicos, muito barulho. E os resultados? Revista Gestão e Planejamento, v. 17, n. 2, p. 298-313, 2016.

VEDOVELLO, C.; JUDICE, V.; MACULAN, A-M. Revisão crítica às abordagens a parques tecnológicos: alternativas interpretativas às experiências brasileiras recentes RAI: Revista de Administração e Inovação, v. 3, n. 2, p. 103-118, 2006.

VIALE, R.; CAMPODALL'ORTO, S. An evolutionary Triple Helix to strengthen academy-industry relations: suggestions from European regions. Science and Public Policy, v. 29, n. 3, p. 154-168, 2002.

VÁZQUEZ B. Desarrollo Endógeno y Globalización. In: MADOERY, O.; VÁZQUEZ BARQUERO, A. Transformaciones globales, instituciones y Politicas de desarrollo local. Rosário: Homo Sapiens, 2001.

VERGARA, S. C. Métodos de pesquisa em administração. São Paulo: Atlas, 2010.

YIN, R. Estudo de caso: planejamento e métodos. 3. ed. Porto Alegre: Bookman, 2005.

ZOUAIN, D. M. Parques tecnológicos - propondo um modelo conceitual para regiões urbanas - o parque tecnológico de São Paulo. 2003. Tese (Doutorado) - Universidade de São Paulo, São Paulo, 2003.

. Recomendações e conclusões do grupo de trabalho. In: HAUSER, G.; ZEN, A. C. Tecnópole: o desafio da sinergia. Porto Alegre: Nova Prova, 2005. p. 17-19. Cap. I. 